Gisele dos Reis Della Togna ${ }^{1}$

Edgard Michel-Crosato ${ }^{1}$

Heloisa Brunow Ventura Di Nubila ${ }^{2}$

Edgard Crosato ${ }^{1}$

\section{Perspectivas de utilização da CIF em saúde bucal do trabalhador}

\author{
Perspectives of using the ICF in worker's oral health
}

\begin{abstract}
${ }^{1}$ Universidade de São Paulo, Faculdade de Odontologia, Departamento de Odontologia Social. São Paulo, SP, Brasil.

${ }^{2}$ Universidade de São Paulo, Faculdade de Saúde Pública, Centro Colaborador da Organização Mundial da Saúde (OMS) para a Família de Classificações Internacionais em Português. São Paulo, SP, Brasil.
\end{abstract}

Contato:

Gisele dos Reis Della Togna

E-mail:

giseletogna@usp.br

Artigo baseado na Dissertação de Gisele dos Reis Della Togna, intitulada Aplicabilidade da CID-10, CID-OE e CIF na análise dos afastamentos do trabalho por motivo odontológico em um serviço público federal, apresentada em 2010, e na Tese da mesma autora, intitulada Utilização da CIF na análise do absenteísmo odontológico, apresentada em 2013, ambas à Faculdade de Odontologia da Universidade de São Paulo.

Este trabalho não foi apresentado em reunião científica.

O trabalho não foi subvencionado.

Os autores declaram não haver conflitos de interesses.

\section{Resumo}

Para a estruturação de um sistema de informação em saúde, é importante o fornecimento de um esquema de codificação. A utilização da Classificação Internacional de Funcionalidade, Incapacidade e Saúde (CIF) permite a descrição da saúde e dos estados relacionados à saúde, avaliando o que é significativo para o indivíduo e complementando a informação sobre o diagnóstico fornecida pela Classificação Estatística Internacional de Doenças e Problemas Relacionados à Saúde - CID. Este ensaio explora a estrutura e o modelo conceitual da CIF, indicando as perspectivas de utilização da CIF em saúde bucal do trabalhador. A avaliação do impacto de uma condição de saúde bucal é fundamental para o planejamento de ações que considerem a integralidade da saúde, bem como sua relação com o bem-estar e a qualidade de vida no trabalho. A CIF é a ferramenta imprescindível para a viabilização de uma abordagem abrangente capaz de abarcar as múltiplas dimensões envolvidas em uma situação de comprometimento da saúde bucal, qualificando as informações disponíveis para a definição de estratégias de intervenção em saúde do trabalhador.

Palavras-chave: Classificação Internacional de Funcionalidade; incapacidade e saúde; saúde do trabalhador; odontologia do trabalho.

\begin{abstract}
A Coding scheme is important for structuring a health information system. The use of the International Classification of Functioning, Disability and Health (ICF) enables the health description and health-related states, evaluating what is meaningful to the individual and complementing the diagnosis information provided by the International Statistical Classification of Diseases and Related Health Problems - ICD. This paper explores the ICF structure and conceptual framework, stating the perspectives of using the ICF in worker's oral health. The impact of oral health assessment is critical for planning actions, considering the health's integrality as well as its relation to the well-being and quality of life at work. The ICF is an essential tool for achieving a comprehensive approach to encompass the multiple dimensions involved in a situation of oral health impairment, qualifying the information available for defining intervention strategies in occupational health.
\end{abstract}

Keywords: International Classification of Functioning; disability and health; occupational health; occupational dentistry. 


\section{Introdução}

A Organização Mundial da Saúde (OMS) destaca a necessidade do desenvolvimento e do fortalecimento de um sistema de informação para a definição de políticas de saúde do trabalhador. A prática e a pesquisa nessa área dependem da disponibilidade de um banco de dados relevante, organizado e de fácil acesso. Nesse sentido, os serviços de saúde do trabalhador devem, regular e sistematicamente, tornar disponível a informação sobre a saúde de uma população, incluindo estatísticas das condições e das necessidades de saúde (WORLD HEALTH ORGANIZATION, 1995).

Para a construção de um sistema de informação em saúde, é importante o fornecimento de um esquema de codificação (ORGANIZAÇÃO MUNDIAL DA SAÚDE, 2008). Por essa razão, o conhecimento aprofundado das classificações internacionais da OMS é fundamental.

Os dados de morbidade são utilizados para o planejamento, o gerenciamento e a avaliação dos programas e políticas de saúde (ORGANIZAÇÃO MUNDIAL DA SAÚDE, 2008). A inclusão dos dados de funcionalidade proporciona um registro completo de saúde, que se mostra essencial para o acompanhamento adequado do trabalhador, gerando uma fonte importante de dados epidemiológicos e qualificando a informação de saúde disponível.

Assim, para uma análise mais profunda do processo saúde-doença-cuidado e para inserir outros dados além da informação sobre o diagnóstico, surgiu o conceito de uma família de classificações de saúde. Esse conjunto de classificações, designado "Família de Classificações da OMS", tem como objetivo prover um modelo conceitual das informações relacionadas à saúde, bem como servir de modelo para a construção dos sistemas de informação em saúde (ORGANIZAÇÃ̃O MUNDIAL DA SAÚDE, 2008).

A Classificação Estatística Internacional de Doenças e Problemas Relacionados à Saúde - CID e a Classificação Internacional de Funcionalidade, Incapacidade e Saúde - CIF são as classificações de referência da "Família de Classificações Internacionais da OMS”. As classificações derivadas são baseadas nas classificações de referência e incluem as adaptações para especialidades, como a Classificação Internacional de Doenças em Odontologia e Estomatologia - CID-OE. A CID-OE relaciona-se com todas as doenças e condições que ocorrem, se manifestam ou estão associadas à cavidade oral e às estruturas adjacentes. Juntas, essas classificações permitem abarcar os aspectos multidimensionais da saúde (ORGANIZAÇÃO MUNDIAL DA SAÚDE, 2008).

A CID e a CIF são consideradas classificações complementares. As informações sobre o diagnóstico e sobre a funcionalidade permitem descrever a saúde das pessoas ou de populações de forma ampla e significativa (ORGANIZAÇÃO MUNDIAL DA SAÚDE, 2003).

Atualmente, a CID é mandatória para a codificação dos dados para os sistemas de informação em mortalidade e morbidade no País.

Alguns conceitos centrados na avaliação subjetiva do indivíduo, tais como qualidade de vida relacionada com a saúde e o estado subjetivo de saúde, estão estreitamente ligados ao impacto de uma condição de saúde sobre a capacidade do indivíduo viver plenamente. Já o termo qualidade de vida envolve uma maior variedade de condições que podem afetar a percepção do indivíduo, seus sentimentos e comportamentos relacionados com a sua funcionalidade diária (REIS; RIBEIRO, 2003).

Importa destacar a necessidade de compreender a doença considerando-se a totalidade do ser humano, permitindo uma tomada de decisão que colabore para a qualidade de vida. Assim, para uma abordagem integrada e humanizada do paciente, é fundamental considerar os parâmetros biológicos, psicológicos, sociais e espirituais (GALLIAN; REGINATO, 2009).

Todos esses aspectos citados relativos à qualidade de vida, ao impacto de uma condição de saúde na funcionalidade diária e às dimensões biopsicossociais estão representados no modelo da CIF.

Nesse contexto, resolução recente do Conselho Nacional de Saúde determina que a CIF seja utilizada no Brasil, Estado Membro da OMS. Essa resolução especifica as possibilidades de utilização da CIF, como, por exemplos: ferramenta estatística na coleta e no registro de dados; ferramenta clínica para avaliar necessidades; uso nas investigações para medir resultados relacionados ao bem-estar, à qualidade de vida, ao acesso aos serviços e ao impacto dos fatores ambientais na saúde dos indivíduos; para o dimensionamento e o redimensionamento dos serviços, visando a obter informações relativas ao tratamento e à recuperação da saúde; ferramenta geradora de informações padronizadas, bem como geradora de indicadores de saúde referentes à funcionalidade humana (BRASIL, 2012).

\section{Classificação Internacional de Funcio- nalidade, Incapacidade e Saúde - CIF}

A CIF foi aprovada na 54. ${ }^{\text {a }}$ Assembleia Mundial da Saúde em maio de 2001 e a versão para o português foi lançada em 2003. O modelo biopsicossocial da CIF, interativo, multidirecional e dinâmico, representa a integração do modelo médico e do modelo social, obtendo-se uma síntese que possibilita uma visão abrangente das várias dimensões de saúde (MARCELINO; DI NUBILA, 2013). 
Nos termos da Resolução WHA54.21, recomenda-se que os Países Membros da OMS utilizem a CIF em suas pesquisas, na vigilância e nos relatórios (WORLD HEALTH ORGANIZATION, 2001).

A CIF classifica a funcionalidade e a incapacidade humana. Ela proporciona uma linguagem unificada e padronizada, bem como uma estrutura para descrever a saúde e os estados relacionados à saúde, definindo os componentes da saúde e alguns componentes do bem-estar relacionado à saúde (ORGANIZAÇÃO MUNDIAL DA SAÚDE, 2003).

A classificação possui 1.424 categorias distribuídas em duas partes (Parte 1 - Funcionalidade e Incapacidade e Parte 2 - Fatores Contextuais) e cada parte tem dois componentes (Parte 1 - componente "Corpo", que inclui "Funções do Corpo" e "Estruturas do Corpo", e componente "Atividades e Participação"; Parte 2 - componente "Fatores Ambientais" e componente "Fatores Pessoais") (MARCELINO; DI NUBILA, 2013).

No modelo proposto pela CIF, a funcionalidade e a incapacidade de uma pessoa são concebidas como uma interação dinâmica entre uma condição de saúde (doenças, distúrbios, lesões, traumas) e os fatores contextuais: ambiental e pessoal (ORGANIZAÇÃO MUNDIAL DA SAÚDE, 2003).

Vale sublinhar que dois indivíduos com um diagnóstico idêntico podem ter diferentes níveis de funcionalidade (ÜSTÜN et al., 2003).

Para descrever a extensão do bem-estar e da qualidade de vida de um indivíduo ou de uma população, é essencial avaliar e descrever a funcionalidade. É importante destacar que a CIF não classifica pessoas, ela descreve, sim, a experiência do indivíduo (CIEZA; BICKENBACH; CHATTERJI, 2008).

Tanto a CID quanto a CIF organizam seus capítulos de acordo com os sistemas do corpo, indicando uma sobreposição entre elas. Contudo, os propósitos das duas classificações são diferentes. Na CID, o foco é o diagnóstico e a classificação etiológica de uma condição de saúde. De modo complementar, a CIF oferece informação sobre o grau de severidade da condição de saúde, permitindo identificar a funcionalidade e a incapacidade associadas à condição de saúde codificada pela CID (ORGANIZAÇÃO MUNDIAL DA SAÚDE, 2003).

A compreensão do modelo conceitual da CIF e sua harmonização com as outras classificações da "Família de Classificações Internacionais da OMS" e com os outros modelos de funcionalidade relacionada à saúde, tais como a promoção da saúde, a qualidade de vida e as atividades da vida diária, podem favorecer sua utilização (SALVADOR-CARULLA; GARCIA-GUTIERREZ, 2011).
Ademais, para facilitar o uso da CIF, a OMS oferece uma lista de itens mínimos para os sistemas de informação em saúde e para as pesquisas (ORGANIZAÇÃO MUNDIAL DA SAÚDE, 2003). Além disso, disponibiliza uma checklist (lista de checagem) com um resumo dos itens da classificação.

A checklist da CIF possui um caráter genérico e, por essa razão, estão sendo desenvolvidos core sets (listas mínimas de itens para grupos ou situações específicas). Atualmente, já foram ou estão sendo desenvolvidos 30 core sets, tais como: dor crônica generalizada, obesidade, diabetes melito, câncer de cabeça e pescoço, e reabilitação profissional. Esses core sets são protocolos validados em consensos internacionais, constituídos de um conjunto mínimo de códigos que oferecem o máximo de informações (MARCELINO; DI NUBILA, 2013).

Um core set específico para avaliação da incapacidade de longo prazo foi desenvolvido com a definição de uma lista de 20 categorias da CIF, sendo 5 do componente "funções do corpo" e 15 do componente "atividades e participação". Esse core set é importante para determinar a elegibilidade a benefícios por incapacidade, auxiliando a tomada de decisões e, com isso, melhorando a qualidade dessas decisões, bem como a comunicação entre profissionais (BRAGE; DONCEEL; FALEZ, 2008).

Em estudo para identificar os problemas mais comuns em pacientes com doenças crônicas, utilizando-se a checklist da CIF, verificou-se que a dor é a única categoria comum a todas as condições crônicas. Os facilitadores mais comuns identificados relacionam-se ao apoio de profissionais de saúde, à atitude dos profissionais de saúde e ao sistema de saúde (EWERT et al., 2004).

A OMS aprovou em 2007 uma adaptação da CIF denominada Classificação Internacional de Funcionalidade, Incapacidade e Saúde - Versão para Crianças e Jovens, a CIF-CJ, com alteração e expansão de algumas descrições, inclusão de códigos, alteração nos critérios de inclusão e exclusão, com o objetivo de retratar as mudanças que ocorrem nas primeiras duas décadas de vida (ORGANIZAÇÃO MUNDIAL DA SAÚDE, 2011).

Vale ressaltar que muitas das categorias adicionais da CIF-CJ podem ser usadas para classificar o nível de funcionalidade de adultos e idosos, como, por exemplo, a categoria d57020 - controlar medicações e seguir aconselhamentos de saúde (MARCELINO; DI NUBILA, 2013).

A CIF serve como uma estrutura para organizar as informações. Suas características estruturais interferem significativamente em seu uso, tais como as definições operacionais padronizadas de seus domínios, 
o sistema alfanumérico e o uso dos qualificadores (ORGANIZAÇÃO MUNDIAL DA SAÚDE, 2003).

Para descrever o perfil de funcionalidade de uma pessoa, pode ser necessária a designação de um conjunto de códigos, observando-se todos os componentes da CIF. Conforme a circunstância em que se realiza o contato, o usuário da classificação pode selecionar os códigos mais relevantes para descrever a experiência de saúde de um indivíduo. Esses códigos devem ser registrados com o maior grau de especificidade possível (ORGANIZAÇÃO MUNDIAL DA SAÚDE, 2003).

No sentido de facilitar a utilização da CIF, a OMS publicou o documento intitulado, na tradução para o português, "Guia para Principiantes - Rumo a uma linguagem comum para Funcionalidade, Incapacidade e Saúde". Esse documento fornece informações sobre os princípios básicos da classificação e sobre como a CIF pode ser usada (ORGANIZAÇÃO MUNDIAL DA SAÚDE, 2002).

O uso da CIF fornece informações relevantes sobre o nível de funcionalidade e incapacidade, sobre o acesso e a qualidade da assistência prestada, bem como sobre os fatores contextuais que influenciam a saúde e a recuperação do indivíduo. Sua abordagem biopsicossocial relaciona a doença ao bem-estar e à qualidade de vida (CIEZA; STUCKI, 2008; FÉRNANDEZ-LÓPEZ; FÉRNANDEZ-FIDALGO; CIEZA, 2010).

Para se estabelecer e facilitar o uso conjunto da CID e da CIF, é necessário um alinhamento de conceitos e terminologias. No processo de revisão em andamento, a CID será complementada com "propriedades funcionais", que são um conjunto de categorias pré-selecionadas e reformuladas pela OMS do componente "Atividades e Participação" da CIF (KOSTANJSEK et al., 2011). Dessa forma, a CID também incluirá o conceito de funcionalidade. Essas propriedades possibilitam selecionar as categorias da CIF mais relevantes para uma determinada condição de saúde (KOHLER et al., 2012).

Na Irlanda, o uso da estrutura conceitual da CIF, no primeiro levantamento nacional de incapacidade, permitiu englobar um maior número de pessoas com incapacidade, além de mostrar que os fatores ambientais na CIF são abrangentes e politicamente relevantes (GOOD, 2011).

Na definição proposta pela CIF, incapacidade indica os aspectos negativos da interação entre um indivíduo (com uma condição de saúde) e seus fatores contextuais (fatores ambientais e pessoais). Incapacidade é um termo genérico para deficiências, limitações de atividade e restrições de participação (ORGANIZAÇÃO MUNDIAL DA SAÚDE, 2003).

Com efeito, uma definição de incapacidade deve incluir todos os aspectos envolvidos na incapacidade (funcionalidade relativa ao corpo, à pessoa e à sociedade), deve destacar a natureza interativa dinâmica da incapacidade e, também, reconhecer o papel da condição de saúde e dos fatores ambientais na produção e na mediação da experiência da incapacidade (LEONARDI et al., 2006).

Concluindo, a CIF identifica as necessidades das pessoas, permitindo a descrição e a classificação do processo saúde-doença-cuidado, aumentando a qualidade e a individualidade dos dados (ORGANIZAÇÃO MUNDIAL DA SAÚDE, 2003), bem como fornecendo informações essenciais para o planejamento de ações em saúde voltadas para as reais necessidades dos indivíduos.

\section{CIF e saúde do trabalhador}

Para a análise das questões relativas à saúde do trabalhador, é fundamental observar as múltiplas dimensões envolvidas no processo de saúde, funcionalidade e incapacidade, considerando a importância do ambiente físico, social e de atitudes (DI NUBILA, 2010).

As informações sobre o estilo de vida e o ambiente de trabalho possibilitam o planejamento de ações de prevenção e promoção da saúde. Vale sublinhar a importância da avaliação da qualidade de vida em saúde do trabalhador, validando os aspectos subjetivos e incluindo a perspectiva do indivíduo (REIS; ARANTES, 2013).

Uma utilização frequente da Classificação Estatística Internacional de Doenças e Problemas Relacionados à Saúde - CID é a sua apresentação em atestados para justificar uma falta ao trabalho (MAZZILLI, 2007).

O uso isolado da CID permite avaliar o perfil de morbidade que gera afastamento do trabalho. Contudo, somente a informação sobre o diagnóstico não fornece a informação necessária para o gerenciamento e o planejamento de saúde (DI NUBILA, 2010). Para isso, obter informações sobre a necessidade de serviços, o nível de cuidados ou os resultados funcionais é fundamental (ORGANIZAÇÃO MUNDIAL DA SAÚDE, 2003). Com o uso complementar da CIF, agregam-se informações sobre a funcionalidade e a incapacidade relacionadas à condição de saúde.

Dentre as possíveis aplicações da CIF em Saúde do Trabalhador, está a avaliação da elegibilidade a benefícios por peritos da Previdência Social (MARCELINO; DI NUBILA, 2013).

Durante o processo de implementação da CIF na Itália, foram desenvolvidos uma checklist da CIF para trabalhadores e um protocolo para avaliação da incapacidade. A checklist da CIF para trabalhadores contém uma seleção de 183 categorias, sendo 89 pertencentes ao domínio de "Atividades e Participação", 
45 do domínio "Funções do Corpo", 16 do domínio "Estruturas do Corpo" e 33 do domínio "Fatores Ambientais". O uso dessa ferramenta baseada na CIF facilita a comunicação e a troca de informações entre empregados e empregadores (CONCLAVE et al., 2009).

$\mathrm{Na}$ avaliação da capacidade para o trabalho, profissionais da seguridade social consideraram importantes os aspectos relacionados às funções e estruturas do corpo, e à participação (SLEBUS et al., 2007). Para os profissionais de reabilitação vocacional, a estrutura conceitual da CIF pode ser utilizada para organizar a informação, fornecendo uma perspectiva abrangente para a recolocação profissional e para o processo de reabilitação (HOMA, 2007).

A CIF pode ser utilizada na avaliação da incapacidade para o trabalho por peritos, contribuindo para incorporar evidência na tomada de decisão. O modelo da CIF serve para definir as perguntas e selecionar os termos em uma estratégia de busca de evidências científicas. Vale ressaltar que a incapacidade para o trabalho é considerada parte do conceito de "participação" da CIF (KOK et al., 2011).

O conceito de participação na CIF refere-se ao envolvimento em uma situação de vida (ORGANIZAÇÃO MUNDIAL DA SAÚDE, 2003). Esse envolvimento, em uma perspectiva ocupacional, pode ser avaliado tanto por um observador externo quanto pelo próprio indivíduo, realçando a experiência subjetiva de significado e autonomia. Nessa perspectiva, é importante considerar a influência dos fatores ambientais que podem ser, ao mesmo tempo, barreiras e facilitadores para a participação (HEMMINGSSON; JONSSON, 2005). Nesse sentido, a identificação das barreiras e dos facilitadores para o retorno ao trabalho, com o uso da CIF, possibilita a definição dos domínios que mais afetam a saúde do trabalhador (TOLDRÁ et al., 2010).

Os peritos podem utilizar várias fontes de informação nas avaliações da incapacidade para o trabalho de longo prazo. Uma fonte de informação é o próprio periciado, que tem a oportunidade de apresentar seus argumentos. Para melhorar a capacidade analítica e a qualidade das avaliações, o perito também pode solicitar informações ao profissional de saúde que prestou a assistência, ao empregador ou a peritos externos. Nessas avaliações, a adesão a protocolos que correspondam aos conceitos e à abordagem biopsicossocial da CIF pode aprimorar a qualidade das avaliações em termos de transparência e reprodutibilidade (DE BOER et al., 2009).

Resumindo, a área de saúde do trabalhador é um dos campos promissores de aplicação da CIF. Por exemplo, a avaliação da incapacidade laborativa por peritos requer informações estratégicas que podem ser obtidas com a utilização do modelo biopsicossocial da CIF. Vale destacar que a compreensão da lógica de codificação é fundamental para qualificar a atuação pericial (MARCELINO; DI NUBILA, 2013).

\section{CIF e saúde bucal}

O Manual dos Usuários da CIF da Austrália indica as referências específicas da classificação para a avaliação de uma condição de saúde bucal e suas consequências (AUSTRALIAN INSTITUTE OF HEALTH AND WELFARE, 2003).

Considerando-se a influência dos valores pessoais e sociais, bem como os múltiplos aspectos envolvidos em uma experiência de saúde bucal, foi proposto, com base nos conceitos e na linguagem da CIF, um novo modelo de avaliação da saúde bucal. Avaliar o que é significativo para o paciente permite obter informações adequadas sobre o impacto de uma condição de saúde bucal para o indivíduo (MACENTEE, 2006). Com efeito, os procedimentos de avaliação da condição de saúde bucal devem considerar esses aspectos para uma intervenção mais adequada.

Em estudo realizado com uma população de idosos, utilizando-se o modelo da CIF, verificou-se que a maioria dos fatores explicativos para a satisfação com a saúde bucal pertencia ao componente "Funções do Corpo": aparência dos dentes, dor de dente, sensação de queimação na boca e mastigação (EKBACK et al., 2012).

Durante trabalhos relacionados à iniciativa de uso conjunto da CID e da CIF, a fenda labial, a fenda palatina e o câncer bucal foram as condições relativas à área odontológica consideradas relevantes para a reabilitação (KOHLER et al., 2012).

Vários estudos utilizam o core set para câncer de cabeça e pescoço na avaliação de resultados de intervenções para tratamento dessa condição de saúde (TSCHIESNER et al., 2012), bem como para avaliar o impacto da doença nas perspectivas do profissional e do paciente (TSCHIESNER et al., 2009; TSCHIESNER; BECKER; CIEZA, 2010; ROGERS et al., 2010).

Para desenvolver um core set da CIF-CJ para saúde bucal, um estudo preliminar identificou as categorias que mais frequentemente apareceram em crianças e jovens com necessidades de cuidado especial. Todos os capítulos do componente "Atividades e participação" foram representados nessa lista. Os facilitadores identificados com maior frequência foram: apoio e atitudes de amigos, família e profissionais de saúde. Por outro lado, a única barreira citada foi a atitude social (FAULKS et al., 2013).

A partir da aplicação de uma seleção de 18 códigos da CIF, estudo realizado em pacientes com deficiência intelectual permitiu identificar os seguintes preditores para tratamento odontológico com anestesia geral: dor na cabeça e pescoço; serviços, sistemas e políticas de transporte, e serviços, sistemas e políticas de educação e treinamento (PETROVIC; MARKOVIC; PERIC, 2011). 
Outro estudo que avaliou pacientes com deficiência intelectual concluiu que a aplicação de, no mínimo, 16 códigos selecionados da CIF pode identificar os preditores para a tolerabilidade ao tratamento odontológico (MAEDA et al., 2005).

Em resumo, observa-se que os estudos supracitados relativos à utilização da CIF na área odontológica referem-se a populações específicas: idosos; pacientes com câncer de cabeça e pescoço, e crianças e adolescentes com deficiência intelectual ou necessidades de cuidados especiais.

Importa sublinhar a importância de implementar o uso da CIF para a coleta de dados nos serviços de saúde do trabalhador para avaliação do impacto de uma condição de saúde bucal na execução das atividades laborativas e na participação no trabalho.

A Norma Regulamentadora n. ${ }^{\circ} 7$ (NR-7) estabelece a obrigatoriedade da elaboração e da implementação, por parte de todos os empregadores e instituições que admitam trabalhadores como empregados, do Programa de Controle Médico de Saúde Ocupacional - PCMSO, com o objetivo de promoção e preservação da saúde do conjunto dos seus trabalhadores. O PCMSO deverá considerar as questões incidentes sobre o indivíduo e a coletividade de trabalhadores, privilegiando o instrumento clínico-epidemiológico na abordagem da relação entre saúde e trabalho, incluindo a realização obrigatória dos exames médicos: admissional, periódico, de retorno ao trabalho, de mudança de função e demissional. Os dados obtidos nesses exames, incluindo a avaliação clínica, os exames complementares, as conclusões e as medidas aplicadas, deverão ser registrados em prontuário clínico individual (BRASIL, 2006).

A inclusão da Odontologia no PCMSO, com a realização de exames odontológicos admissionais e periódicos, além de incluir a saúde bucal no sistema de saúde ocupacional, permitiria a criação de um banco de dados para a área odontológica (ARAÚJO; GONINI JÚNIOR, 1999). Vale sublinhar que essa necessidade refere-se também aos programas de atenção à saúde dos trabalhadores estatutários, alcançando, desse modo, toda a classe trabalhadora do País.

Como os dados epidemiológicos são de importância fundamental para o planejamento de programas de saúde bucal do trabalhador, a implantação de um sistema eficaz de informação permitirá estabelecer mecanismos de avaliação contínua dos programas de saúde bucal com base nos princípios da promoção da saúde, na redução da incidência das doenças bucais, bem como das necessidades de tratamento odontológico (ALMEIDA; VIANNA, 2005).

Em estudo de 1999, verificou-se que não se fazia, à época, uma Odontologia do Trabalho voltada para a identificação epidemiológica, a catalogação ou a prevenção das doenças e sim voltada para o aspecto curativo. Uma abordagem epidemiológica permite estudar o impacto que uma condição de saúde bucal pode causar à qualidade de vida dos trabalhadores (ARAÚJO; GONINI JÚNIOR, 1999). No momento atual, essas questões devem ser consideradas na organização dos serviços de saúde do trabalhador, com ações integradas e a inclusão da avaliação da saúde bucal.

Em relação à incapacidade para o trabalho por motivo odontológico, vale ressaltar que uma mesma condição de saúde bucal pode exigir períodos de afastamentos do trabalho diferenciados e o uso isolado da CID não permite um aprofundamento na análise dessas diferenças, que poderiam ser investigadas e registradas com o uso complementar da CID-OE e da CIF. Sobre esse aspecto, ainda é necessário aperfeiçoar a utilização da CID e incentivar o uso da CID-OE entre os profissionais de Odontologia (TOGNA et al., 2011), incluindo o uso complementar da CIF, qualificando a análise das questões relativas à saúde bucal do trabalhador.

A avaliação do nível de funcionalidade e incapacidade associadas a uma condição de saúde bucal orienta a prática do profissional de Odontologia nos serviços de saúde do trabalhador. Por exemplo, no caso da perda de um elemento dentário, o uso da CIF torna possível avaliar o impacto dessa condição de saúde bucal, observando-se as funções alteradas (sugar, morder, mastigar, dor, funções emocionais); as atividades e participação afetadas (falar, comer, cuidado dos dentes, necessidade de afastamento do trabalho), bem como os fatores ambientais que atuam como facilitadores (medicamentos, alimentos) ou barreiras (acesso ao serviço de saúde bucal) para a recuperação da saúde.

Ademais, a utilização da CIF aportaria informações relevantes sobre a qualidade da assistência prestada, bem como sobre os fatores contextuais que influenciam a saúde e a recuperação do indivíduo, melhorando a funcionalidade e reduzindo a incapacidade.

Com o uso da CIF, a identificação dos fatores ambientais que afetam a condição de saúde bucal de um indivíduo pode favorecer a definição de áreas prioritárias para o planejamento de ações de educação em saúde bucal no trabalho.

Na medida em que a CIF pode ser utilizada para descrever a experiência de saúde de qualquer pessoa, não somente de pessoas com incapacidades (ORGANIZAÇÃO MUNDIAL DA SAÚDE, 2003), é possível considerar sua aplicação nos exames admissionais e periódicos, contribuindo para o acompanhamento da situação de saúde durante o período de vida laborativa de um indivíduo, bem como favorecendo a proteção e a promoção da saúde desse trabalhador. 
A inclusão dos exames ocupacionais odontológicos e o registro dos dados coletados com o uso conjunto da CID e da CIF podem contribuir para o monitoramento da saúde bucal do trabalhador, relacionando-a com a saúde geral, o bem-estar e a qualidade de vida no trabalho.

\section{Considerações finais}

O uso das classificações internacionais da OMS confere qualidade ao registro das informações em saúde. A disponibilidade de dados corretos sobre o perfil do adoecimento e de funcionalidade dos trabalhadores é fundamental para o planejamento de ações em saúde bucal do trabalhador.

Logo, a qualidade da informação disponível é essencial para a tomada de decisões que orientam a prática. É importante para a promoção da saúde e para melhorar a prevenção de agravos e a organização dos serviços de saúde do trabalhador.
O entendimento da complexidade e do dinamismo das questões relativas à saúde geral e à saúde bucal exige uma visão mais abrangente da saúde. O modelo conceitual da CIF propicia essa abordagem, permitindo compreender o ser humano em sua totalidade e singularidade. Esse novo olhar tem uma importância significativa para a definição de estratégias que determinam as ações de saúde a serem implantadas, no sentido de ampliar as capacidades e estimular o desenvolvimento pleno das potencialidades de cada trabalhador.

A CIF fornece dados que, agregados aos dados de morbidade gerados pela CID e pela CID-OE, podem determinar as medidas de intervenção em saúde que favoreçam o bem-estar e a qualidade de vida.

No contexto atual, é imprescindível o conhecimento da estrutura e dos propósitos das classificações, aprimorando o padrão de utilização da CID e da CID-OE, assim como incorporando a utilização da CIF na análise das questões relativas à saúde bucal do trabalhador.

\section{Contribuições de autoria}

Togna, G. R. D.: elaboração do manuscrito. Michel-Crosato, E., Di Nubila, H. B. e Crosato, E.: revisão crítica e aprovação final do manuscrito.

\section{Referências}

ALMEIDA, T. F.; VIANNA, M. I. P. O papel de epidemiologia no planejamento das ações de saúde bucal do trabalhador. Saúde e Sociedade, São Paulo, v. 14, n. 3, p. 144-154, 2005. http://dx.doi.org/10.1590/ S0104-12902005000300010.

ARAÚJO, M. E.; GONINI JÚNIOR, A. Saúde bucal do trabalhador: os exames admissional e periódico como um sistema de informação em saúde. Odontologia e Sociedade, São Paulo, v. 1, n. 1-2, p. 15-18, 1999.

\section{AUSTRALIAN INSTITUTE OF HEALTH AND} WELFARE - AIHW. ICF Australian user guide version 1.0. Canberra: AIHW, 2003. Disability Series. Cat., DIS 33. Disponível em: <https://www.aihw.gov.au/ WorkArea/DownloadAsset.aspx?id =6442455729 > . Acesso em: 28 jan. 2014.

BRAGE, S.; DONCEEL, P.; FALEZ, F. Development of ICF core set for disability evaluation in social security. Disability and Rehabilitation, London, v. 30, n. 18, p. 1392-1396, 2008. http://dx.doi. org/10.1080/09638280701642950. PMid:18850352.

BRASIL. Segurança e medicina do trabalho: lei $\mathrm{n}^{\circ}$ 6.514, de 22 de dezembro de 1977. 59. ed. São Paulo: Atlas, 2006.
. Ministério da Saúde. Resolução no 452 de 10 de maio de 2012. Diário Oficial [da] República Federativa do Brasil, Brasília, DF, 6 jun. 2012. Disponível em: <http://bvsms.saude.gov.br/bvs/ saudelegis/cns/2012/res0452_10_05_2012.html >. Acesso em: 13 ago. 2013.

CIEZA, A.; BICKENBACH, J.; CHATTERJI, S. The ICF as a conceptual plataform to specify and discuss health and health-related concepts. Gesundheitswesen, Stuttgart, v. 70, n. 10, p. e47-e56, 2008. http://dx.doi. org/10.1055/s-2008-1080933. PMid:18932116.

CIEZA, A.; STUCKI, G. The International Classification of Functioning Disability and Health: its development process and content validity. European Journal of Physical and Rehabilitation Medicine, Torino, v. 44, n. 3, p. 303-313, 2008. PMid:18762740.

CONCLAVE, M. et al. The ICF and Labour Policies Project: the first Italian nation-wide experience of ICF implementation in the labour sector. Disability and Rehabilitation, London, v. 31, p. S16-S21, 2009. Suplemento 1. http://dx.doi. org/10.3109/09638280903317823. PMid:19968530.

DE BOER, W. E. et al. Interviews for the assessment of long-term incapacity for work: a study on 
adherence to protocols and principles. BMC Public Health, London, v. 2, n. 9, p. 169, 2009. http://dx.doi. org/10.1186/1471-2458-9-169. PMid:19490614.

DI NUBILA, H. B. V. Uma introdução à CIF: Classificação Internacional de Funcionalidade, Incapacidade e Saúde. Revista Brasileira de Saúde Ocupacional, São Paulo, v. 35, n. 121, p. 122-123, 2010. http://dx.doi.org/10.1590/S030376572010000100013.

EKBACK, G. et al. Oral health of 65-year old in Sweden and Norway: a global question and ICF, the latest conceptual model from WHO. Acta Odontologica Scandinavica, Stockholm, v. 70, n. 4, p. 279-288, 2012. http://dx.doi.org/10.3109/00016357.2011.647069 . PMid:22243522.

EWERT, T. et al. Identification of the most common patient problems in patients with chronic conditions using ICF checklist. Journal of Rehabilitation Medicine, London, v. 44, n. 44, p. 22-29, 2004. Suplemento. http://dx.doi. org/10.1080/16501960410015362. PMid:15370744.

FAULKS, D. et al. Using the International Classification of Functioning, Disability and health (ICF) to describe children referred to special care or pediatric dental services. PLoS One, San Francisco, v. 8, n. 4, p. e61993, 2013. http://dx.doi.org/10.1371/ journal.pone.0061993. PMid:23614000.

FÉRNANDEZ-LÓPEZ, J. A.; FÉRNANDEZ-FIDALGO, M.; CIEZA, A. Los conceptos de calidad de vida, salud y bienestar analizados desde la perspectiva de la Classificacíon Internacional del Funcionamiento (CIF). Revista Española de Salud Pública, Madrid, v. 84, n. 2, p. 169-184, 2010.

GALLIAN, D. M. C.; REGINATO, V. Relação assistencial e sua humanização. In: RAMOS, D. L. P. Bioética: pessoa e vida. São Caetano do Sul: Difusão, 2009. cap. 7, p. 117-133.

GOOD, A. Using the ICF in Ireland. BMC Public Health, London, v. 11, p. S5, 2011. Suplemento 4. http://dx.doi.org/10.1186/1471-2458-11-S4-S5. PMid:21624191.

HEMMINGSSON, H.; JONSSON, H. An occupational perspective on the concept of participation in the International Classification of Functioning Disability and Health: some critical remarks. American Journal of Occupational Therapy, New York, v. 59, n. 5, p. 569-576, 2005. http://dx.doi.org/10.5014/ajot.59.5.569. PMid:16268024.

HOMA, D. B. Using the International Classification of Functioning, Disability and Health (ICF) in job placement. Work, Reading, v. 29, n. 4, p. 277-286, 2007. PMid:18057567.

KOHLER, F. et al. Toward the joint use of ICD and ICF: a call for contribution. Journal of Rehabilitation Medicine, Stockholm, v. 44, n. 10, p. 805-810,
2012. http://dx.doi.org/10.2340/16501977-1062. PMid:22990383.

KOK, R. et al. Integrating evidence in disability evaluation by social insurance physicians.

Scandinavian Journal of Work, Environment \& Health, Helsinki, v. 37, n. 6, p. 494-501, 2011.

KOSTANJSEK, N. et al. Assessing the impact of health conditions using the ICF. Disability and Rehabilitation, London, v. 33, n. 15-16, p. 1475-1482, 2011. http://dx.doi.org/10.3109/09638288.2010.527032 . PMid:20946009.

LEONARDI, M. et al. The definition of disability: what is in a name? The Lancet, London, v. 368, n. 9543, p. 1219-1221, 2006. http://dx.doi.org/10.1016/S01406736(06)69498-1. PMid:17027711.

MACENTEE, M. I. An existential model of oral health from evolving views on health, function and disability. Community Dental Health, London, v. 23, n. 1, p. 5-14, 2006. PMid:16555713.

MAEDA, S. et al. Assessment of patients with intellectual disability using the International Classification of Functioning, Disability and Health to evaluate dental treatment tolerability. Journal of Intellectual Disability Research, Oxford, v. 49, n. 4, p. 253-259, 2005. http://dx.doi.org/10.1111/j.13652788.2005.00644.x. PMid:15816812.

MARCELINO, M. A.; DI NUBILA, H. B. V. A Classificação Internacional de Funcionalidade, Incapacidade e Saúde (CIF) e potenciais aplicações em Saúde do Trabalhador. In: MENDES, R. Patologia do trabalho. 3. ed. São Paulo: Atheneu, 2013. cap. 9. p. 293-324.

MAZZILLI, L. E. N. Odontologia do trabalho. 2. ed. São Paulo: Livraria Santos, 2007.

ORGANIZAÇÃO MUNDIAL DA SAÚDE - OMS. Rumo a uma linguagem comum para Funcionalidade, Incapacidade e Saúde - CIF. Genebra: OMS, 2002. Disponível em: < http://www.fsp.usp.br/ cbcd/ Material/Guia_para_principiantes_CIF_cbcd.pdf $>$. Acesso em: 28 jan. 2014.

. Classificação internacional de funcionalidade, incapacidade e saúde. São Paulo: Edusp, 2003.

. Classificação estatística internacional de doenças e problemas relacionados à saúde: décima revisão: manual de instrução. 8. ed. rev. ampl. São Paulo: Edusp, 2008. v. 2.

. Classificação internacional de funcionalidade, incapacidade e saúde: versão para crianças e jovens. São Paulo: Edusp, 2011.

PETROVIC, B.; MARKOVIC, D.; PERIC, T. Evaluating the population with intellectual disability to comply routine dental treatment using the International Classification of Functioning, Disability and Health. Disability and Rehabilitation, London, v. 33, n. 19-20, 
p. 1746-1754, 2011. http://dx.doi.org/10.3109/0963828 8.2010.546934. PMid:21859419.

REIS, P.; ARANTES, E. F. Contribuição da gestão da informação em saúde para a promoção da saúde e prevenção das doenças. In: MENDES, R. Patologia do trabalho. 3. ed. São Paulo: Atheneu, 2013. cap. 54. p. 1726-1778.

REIS, P.; RIBEIRO, P. C. L. Detecção de agravos à saúde relacionados com o trabalho e o uso gerencial da informação. In: MENDES, R. Patologia do trabalho. 2. ed. Rio de Janeiro: Atheneu, 2003. cap. 6. p. 231-322.

ROGERS, S. N. et al. Development of the Classification of Functioning, Disability and Health as a brief head and neck cancer patient questionaire. International Journal of Oral \& Maxillofacial Surgery, Copenhagen, v. 39, n. 10, p. 975-982, 2010. http:// dx.doi.org/10.1016/j.ijom.2010.06.006.

SALVADOR-CARULLA, N.; GARCIA-GUTIERREZ, C. The WHO construct of health-related functioning $(\mathrm{HrF})$ and its implications for health policy. $B M C$ Public Health, London, v. 11, p. S9, 2011. Suplemento 4. http://dx.doi.org/10.1186/1471-2458-11-S4-S9. PMid:21624195.

SLEBUS, F. G. et al. Work-ability evaluation: a piece of cake or a hard nut to crack? Disability and Rehabilitation, London, v. 29, n. 16, p. 1295-1300, 2007. http://dx.doi.org/10.1080/09638280600976111. PMid:17654004.

TOGNA, G. R. D. et al. Uso da Classificação Internacional de Doenças na análise do absenteísmo odontológico. Revista de Saúde Pública, São Paulo, v. 45, n. 3, p. 512-518, 2011. http://dx.doi.org/10.1590/ S0034-89102011000300009.

TOLDRÁ, R. C. et al. Facilitadores e barreiras para o retorno ao trabalho: a experiência de trabalhadores atendidos em um Centro de Referência em Saúde do Trabalhador - SP, Brasil. Revista Brasileira de Saúde Ocupacional, São Paulo, v. 35, n. 121, p. 10-22, 2010. http://dx.doi.org/10.1590/S0303-76572010000100003.

TSCHIESNER, U. et al. Evaluating sequelae after head and neck cancer from the patient perspective with the help of the internacional classification of functioning, disability and health. European Archives of Otorhinolaryngology, Berlin, v. 266, n. 3, p. 425-436, 2009. http://dx.doi.org/10.1007/s00405-008-0764-z. PMid:18726562.

. Functional outcome in patients with advanced head and neck cancer: surgery and reconstruction with free flaps versus primary radiochemotherapy. European Archives of Otorhinolaryngology, Berlin, v. 269, n. 2, p. 629-638, 2012. http://dx.doi.org/10.1007/ s00405-011-1642-7. PMid:21643935.

TSCHIESNER, U.; BECKER, S.; CIEZA, A. Health professional perspective on disability in head and neck cancer. Archives of Otolaryngology Head \& Neck Surgery, Chicago, v. 136, n. 6, p. 576-583, 2010. http:// dx.doi.org/10.1001/archoto.2010.78. PMid:20566908.

ÜSTÜN, T. B. et al. WHO's ICF and functional status information in health records. Health Care Financing Review, Baltimore, v. 24, n. 3, p. 77-88, 2003. PMid:12894636.

WORLD HEALTH ORGANIZATION - WHO. Global strategy on occupational health for all. Geneva: WHO, 1995. Disponível em: < http://www.who.int/ occupational_health/en/oehstrategy.pdf $>$. Acesso em: 15 out. 2013.

Fifty-Fourth World Health Assembly. Geneva: WHO, 2001. Disponível em: < http://www.who.int/ classifications/icf/wha-en.pdf $>$. Acesso em: 15 set. 2013. 